\title{
Crystalline structure and molecular mobility of PVDF chains in PVDF/PMMA blend films analyzed by solid-state ${ }^{19} \mathrm{~F}$ MAS NMR spectroscopy
}

\author{
Yu Koseki, Keitaro Aimi and Shinji Ando
}

The crystalline structure and molecular mobility of poly(vinylidene fluoride) (PVDF) blended with poly(methyl methacrylate) (PMMA) have been investigated using solid-state ${ }^{19} \mathrm{~F}$ magic angle spinning (MAS) NMR spectroscopy. The origin of the characteristic crystalline peaks observed for three typical polymorphs of PVDF ( $\alpha$-, $\beta$ - and $\gamma$-forms) are explained based on the density functional theory (DFT) calculations of a model compound. Variations in the crystalline conformation of PVDF chains in the blends, the degree of crystallinity and the molecular mobility in the amorphous phase are quantitatively analyzed using the NMR spectral shapes and the ${ }^{19} \mathrm{~F}$ spin-lattice relaxation time in the rotating frame $\left(T_{1 \rho}{ }^{\mathrm{F}}\right)$. The dominant crystalline form in the blends of $[P V D F / P M M A]=[80 / 20],[70 / 20]$ and $[50 / 50]$ is the $\alpha$-form containing a small amount of the $\beta$-form, whereas films made with a [65/35], [60/40] and [55/45] ratio consist of the $\beta$ - and $\gamma$-form. This finding indicates that PVDF chains possess more elongated conformations along their polymer chains in the latter blends. In contrast, no crystalline structure is found in [45/55], [40/60] and [20/80], which indicates that PVDF and PMMA chains are miscible at the molecular level. A minimum value of $T_{1 \rho} \mathrm{F}$ is observed for [60/40], and the $T_{1 \rho} \mathrm{F}$ value increases as the PVDF fraction decreases. The restricted molecular motion in the amorphous phase at lower PVDF fractions with elevated $T_{g} \mathrm{~s}$ is reflected in the increase in spectral widths and the $T_{1 \rho}{ }^{\mathrm{F}}$ values.

Polymer Journal (2012) 44, 757-763; doi:10.1038/pj.2012.76; published online 16 May 2012

Keywords: ${ }^{19}$ F MAS NMR; PMMA; polymer blend; polymorph; PVDF; solid-state NMR

\section{INTRODUCTION}

Poly(vinylidene fluoride) (PVDF), having a repeating unit of $-\left(\mathrm{CH}_{2}-\mathrm{CF}_{2}\right)-$, has been reported to possess several crystalline polymorphs $\left(\alpha-, \beta-, \quad \gamma-, \delta\right.$-, $\varepsilon$-forms).$^{1-4}$ The main chain conformations of the $\alpha$-, $\beta$ - and $\gamma$-forms are $\operatorname{tg}^{+} \operatorname{tg}^{-}$, all-trans (tttt), $\mathrm{tttg}^{+} \mathrm{tttg}^{-}$, respectively. The melting temperatures $\left(T_{\mathrm{m}}\right)$ vary in the range of $170-190^{\circ} \mathrm{C}$ depending on the crystalline polymorphs. The glass transition temperature $\left(T_{\mathrm{g}}\right)$ is below $-35^{\circ} \mathrm{C}$, indicating that the amorphous phase is in the rubbery state at ambient temperatures. Wang and Nishi ${ }^{5,6}$ have reported that semi-crystalline PVDF is compatible with amorphous poly(methyl methacrylate) (PMMA) in the molten state, although PVDF forms crystallites in blends when the weight fraction of PVDF is high. With wide-angle X-ray diffraction and FT-IR spectroscopy, Horinabe and $\mathrm{Baba}^{7}$ investigated the PVDF crystalline structures in PVDF/PMMA blends at variable weight fractions with different thermal treatments. They reported that PVDF component crystallized in the $\beta$-form when the weight fractions of the blend are between $[\mathrm{PVDF} / \mathrm{PMMA}]=[70 / 30]$ and [80/20]. Furthermore, several researchers have investigated the morphology of PVDF and PVDF/PMMA blends using differential scanning calorimetry (DSC), FT-IR, X-ray diffraction, and solid-state
${ }^{13} \mathrm{C}$ CP/MAS NMR. ${ }^{8-11}$ These techniques, however, have difficulties investigating the detailed morphology and molecular mobility of PVDF and PVDF/PMMA blends on the molecular level. For instance, the IR absorptions and wide-angle X-ray diffraction peaks from PVDF crystallites are, in general, overlapped by the peaks from amorphous PMMA. In particular, it is nontrivial to distinguish the crystalline structure of the $\beta$-form from the $\gamma$-form because both are elongated chain conformations.

Solid-state ${ }^{19} \mathrm{~F}$ magic angle spinning (MAS) NMR has been a versatile tool for investigating the morphology of fluorine-containing polymers, including the degree of crystallization, the conformation of crystalline forms and local and global molecular motion. The high natural abundance of ${ }^{19} \mathrm{~F}$ nuclei $(100 \%)$ and the high magnetogyric ratio $\left(94 \%\right.$ of $\left.{ }^{1} \mathrm{H}\right)$ are advantageous to the solid-state ${ }^{19} \mathrm{~F}$ MAS NMR measurements. In addition, ${ }^{19} \mathrm{~F}$ MAS NMR spectrum can be investigated in detail owing to the large chemical shift range (>200 p.p.m.) of the ${ }^{19} \mathrm{~F}$ nuclei. ${ }^{12-14}$ Holstein et al. ${ }^{15,16}$ have investigated the polymorphism in PVDF using ${ }^{1} \mathrm{H}$-decoupled solidstate ${ }^{19} \mathrm{~F}$ MAS NMR. One PVDF sample, prepared through biaxial drawing of a film, mainly contained $\beta$-form, other samples, prepared by melt-recrystallization, mainly contained $\alpha$-form. They reported 
that the crystalline $\alpha$-form PVDF has two peaks with the same signal intensity at $\delta_{\mathrm{F}}=-82$ and -98 p.p.m., whereas the crystalline $\beta$-form of PVDF has a single peak at $\delta_{\mathrm{F}}=-98$ p.p.m. for its crystalline domain. The differences between the spectra of the $\alpha$ - and $\beta$-forms were well explained based on chain conformation, taking into account the well-known $\gamma$-gauche shielding effect. All the fluorines in an alltrans $\beta$-form are equivalent and have two $\gamma$-gauche interactions with carbons, whereas the fluorines are non-equivalent for the $\alpha$-form and, because of its $\mathrm{tg}^{+} \mathrm{tg}^{-}$conformation, one of these two fluorines has one $\gamma$-gauche and one $\gamma$-trans carbon. The chemical shift of the $\beta$-form is thus expected to nearly coincide with one of those of the $\alpha$-form. The present authors have investigated solid-state ${ }^{1} \mathrm{H} \rightarrow{ }^{19} \mathrm{~F} /$ ${ }^{19} \mathrm{~F} \rightarrow{ }^{1} \mathrm{H}$ CP/MAS NMR spectra of PVDF with ${ }^{1} \mathrm{H} /{ }^{19} \mathrm{~F}$ decoupling. Significant differences in $T_{1 \rho}{ }^{\mathrm{F}}$ and $T_{1 \rho}{ }^{\mathrm{H}}$ between the crystalline and amorphous domains were confirmed by spin-lock experiments in the ${ }^{19} \mathrm{~F}$ channel under high-resolution conditions. ${ }^{17,18}$ In addition, we have investigated the molecular structure and dynamics of a vinylidene fluoride oligomer telomerized by carbon tetrachloride (Cl-OVDF) and its inclusion compound $\beta$-cyclodextrin using ${ }^{1} \mathrm{H} \rightarrow$ ${ }^{19} \mathrm{~F}$ CP/MAS NMR. ${ }^{19}$ The crystalline-selective ${ }^{19} \mathrm{~F}$ CP/MAS spectra observed for a refined Cl-OVDF with a larger molecular weight agrees well with the MAS spectrum of PVDF in the $\gamma$-form reported by Hucher et al. ${ }^{20}$ Therefore, the major crystalline polymorph in the sample was identified as $\gamma$-form $\left(\mathrm{ttg}^{+} \mathrm{tttg}^{-}\right)$.

Veeman and coworkers ${ }^{10,11}$ investigated the scale of miscibility in PVDF/PMMA blends using ${ }^{19} \mathrm{~F} \rightarrow{ }^{1} \mathrm{H} \rightarrow{ }^{13} \mathrm{C}$ double cross polarization triple resonance NMR experiments. They suggested that the average distance between the $\mathrm{C}=\mathrm{O}$ or $\mathrm{OCH}_{3}$ carbon of PMMA and the PVDF fluorines is approximately $30 \mathrm{~nm}$ in miscible blends. Furthermore, the present authors have analyzed the crystalline structures of PVDF in PVDF/silica $\left(\mathrm{SiO}_{2}\right)$ binary and $\mathrm{PVDF} / \mathrm{SiO}_{2} /$ PMMA ternary hybrid composite films using ${ }^{19} \mathrm{~F}$ MAS NMR. ${ }^{21}$ The observed seven signals were attributed to the amorphous domain ( -88 p.p.m.), the crystalline domains $(-101.3,-93.7,-84.2$ and -79.6 p.p.m. $)$ and the regio-irregular structures $(-112.4$ and -110.4 p.p.m.). The ${ }^{19} \mathrm{~F}$ MAS spectra of the hybrid composite films showed two resonances assignable to the $\gamma$-form at lower $(-101.3$ p.p.m. $)$ and higher (-84.2 p.p.m.) frequencies. These signals demonstrated long ${ }^{19} \mathrm{~F}$ spin-lattice relaxation times in the rotating frame $\left(T_{1 \rho}{ }^{\mathrm{F}}>20 \mathrm{~ms}\right)$, and their chemical shifts significantly differ from those of the $\alpha$ - and $\beta$-forms.

In this paper, the crystalline structure, degree of crystallinity and the local molecular mobility in the amorphous phase of PVDF chains included in a series of PVDF/PMMA blend films have been investigated from the spectral shapes and the relaxation time $\left(T_{1 \rho}{ }^{\mathrm{F}}\right)$ obtained from ${ }^{19} \mathrm{~F}$ MAS NMR spectra with the aid of quantum chemical calculations of nuclear magnetic shielding.

\section{EXPERIMENTAL PROCEDURE}

\section{Preparation of PVDF films}

PVDF $\left(\right.$ KF-850; $\left.M_{\mathrm{w}}=30.7 \times 10^{4}\right)$ was obtained from Kureha Chemical Industry Co., Ltd. (Tokyo, Japan) and atactic PMMA $\left(M_{\mathrm{w}}=120000\right)$ was purchased from Aldrich (Milwaukee, WI, USA). PVDF pellets were pulverized into a granular powder using a fracture machine to facilitate MAS spinning. The powder contains $\alpha$-form crystallites as the major component because it is the most stable conformation in melt-crystallized PVDF. The characteristic IR bands at $976,855,795$ and $766 \mathrm{~cm}^{-1}$ are all assignable to the $\alpha$-form. This sample will be called ' $\alpha$-powder'. A Kureha KF Piezo Film ( $\beta$-film; Kureha, Tokyo, Japan) containing $\beta$-form crystallites as the major component was prepared through the uniaxial drawing of a PVDF film containing $\alpha$-form crystallites and applying a high-voltage poling to a film in which the crystal structures have an all-trans $\beta$-form conformation. The characteristic IR bands at 840,511 and $470 \mathrm{~cm}^{-1}$ are all assignable to the $\beta$-phase. A PVDF film containing $\gamma$-form crystallites as the major component $(\gamma$-film) was prepared by dissolving PVDF powder in $\mathrm{N}, \mathrm{N}$-dimethylformamide, casting the solution on a plate, annealing at $170{ }^{\circ} \mathrm{C}$ for $12 \mathrm{~h},{ }^{21}$ followed by cooling at a standard rate (for example, $10-20^{\circ} \mathrm{C} \mathrm{min}^{-1}$ ). The characteristic IR bands at 835,510 and $430 \mathrm{~cm}^{-1}$ are all assignable to the $\gamma$-phase, although a certain amount of $\beta$ form $\left(470 \mathrm{~cm}^{-1}\right)$ is included. These samples ( $\alpha$-powder, $\beta$-film and $\gamma$-film) were prepared as reference samples, which were used to estimate the chain conformation generated in the PVDF/PMMA blend films.

\section{Preparation of PVDF/PMMA blends}

$\mathrm{N}, \mathrm{N}$-dimethylacetoamide (DMAc) was purchased from Kanto Chemical Industry Co., Ltd. (Tokyo, Japan). PVDF and PMMA pellets were dissolved in DMAc to make polymer solutions with a total concentration of $25 \mathrm{wt} \%$. Ten different weight fraction blends were used to make various polymer solutions, PVDF/PMMA $=100: 0,80: 20,70: 30,65: 35,60: 40,55: 45,50: 50,45: 55,40,60$ and 20:80 were prepared. Blended films were obtained by spin coating the various solutions onto silicon substrates followed by drying at $70^{\circ} \mathrm{C}$ under atmospheric pressure for 1 day followed by 2 days under vacuum..$^{22}$ To enhance the compatibility between PVDF and PMMA on the molecular level, these films were annealed at $200^{\circ} \mathrm{C}$ for $5 \mathrm{~min}$ and then quenched to $0^{\circ} \mathrm{C}$ through immersion in ice water. In this study, a blend sample of PVDF:PMMA $=\mathrm{X}:(100-\mathrm{X})(\mathrm{wt} \%)$ is denoted as $[\mathrm{X} / 100-\mathrm{X}]$.

\section{Solid-state ${ }^{19}$ F MAS NMR measurements}

Solid-state ${ }^{19} \mathrm{~F}$ MAS NMR was performed on a JEOL EX spectrometer (JEOL, Tokyo, Japan) operating at $282.65 \mathrm{MHz}$ for ${ }^{19} \mathrm{~F}$ and $300.4 \mathrm{MHz}$ for ${ }^{1} \mathrm{H}$, respectively, with a Chemagnetics ${ }^{1} \mathrm{H}-{ }^{19} \mathrm{~F}$ double-tuned APEX MAS probe. The samples were spun at the magic angle at a rate of $16 \mathrm{kHz}$. Because of frictional heat attributed to the high-speed MAS, the ambient probe temperature was $68{ }^{\circ} \mathrm{C}$. The $\pi / 2$ pulse for ${ }^{19} \mathrm{~F}$ nuclei was $3.0 \mu \mathrm{s}$ (ca. $83 \mathrm{kHz}$ ), and ${ }^{1} \mathrm{H}$ decoupling fields was ca. $68 \mathrm{kHz}$. The number of transients acquired for each spectrum was 32 or 64 times, and the recycle delay time was $5.0 \mathrm{~s}$. In the ${ }^{19} \mathrm{~F}$ spin-lattice relaxation time rotating frame $\left(T_{1 \rho}{ }^{\mathrm{F}}\right)$ measurement, the ${ }^{19} \mathrm{~F}$ magnetization is spin locked for a variable spin-lock time, $t_{\mathrm{SL}}$, prior to signal acquisition. Chemical shifts are quoted with respect to the signal for $\mathrm{CFCl}_{3}$ (0 p.p.m.) as an external standard. To correct Bloch-Siegert shift, liquid $\mathrm{C}_{6} \mathrm{~F}_{6}$ $\left(-163.6\right.$ p.p.m.) was measured with ${ }^{1} \mathrm{H}$ decoupling $(68 \mathrm{kHz}){ }^{23}$

\section{DSC measurements}

Glass transition temperatures $\left(T_{\mathrm{g}}\right)$ of the blends were determined using a differential scanning calorimeter (SEIKO Instruments, Tokyo, Japan, DSC220). The measured temperature range was between -80 and $200{ }^{\circ} \mathrm{C}$ with a heating rate of $10^{\circ} \mathrm{C} \mathrm{min}^{-1}$.

\section{Magnetic shielding calculation}

A model compound containing 8-mers of the VDF sequence, $\mathrm{CH}_{3} \mathrm{CF}_{2}-$ $\left(\mathrm{CH}_{2}-\mathrm{CF}_{2}\right)_{7}-\mathrm{CH}_{3}$, was adopted for the ${ }^{19} \mathrm{~F}$ nuclear magnetic shielding calculation. Density functional theory (DFT) calculations were performed using the Gaussian-09 B.01 software package in which B3LYP/6-311G(d) was used for geometry optimization and B3LYP/6-311 $+\mathrm{G}(2 \mathrm{~d}, \mathrm{p})$ was used for the magnetic shielding calculations.

\section{RESULTS AND DISCUSSION}

\section{PVDF crystalline structures in reference samples}

Figure 1 shows the solid-state ${ }^{19}$ F MAS NMR spectra of the $\alpha$-powder, $\beta$-film and $\gamma$-film of PVDF. Spectra (a)-(c) were observed using a direct polarization (DP) pulse sequence with broadband ${ }^{1} \mathrm{H}$ decoupling (DP), and spectra (d)-(f) were observed using the crystallineselective pulse sequence with spin locking (SL) for $20 \mathrm{~ms}$. Each spectrum can be fitted by a combination of Lorentzian broadening functions. ${ }^{18,19}$ In the DP spectrum of the $\alpha$-powder (Figure 1a) the peaks at $\delta_{\mathrm{F}}=-79.4(\mathrm{~A})$ and -93.7 p.p.m. (B) are assigned to the crystalline domains, the peak at $\delta_{\mathrm{F}}=-88.7$ p.p.m. (C) is assigned 
a

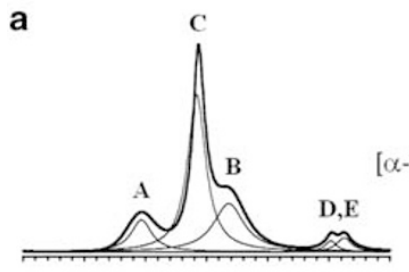

d

b

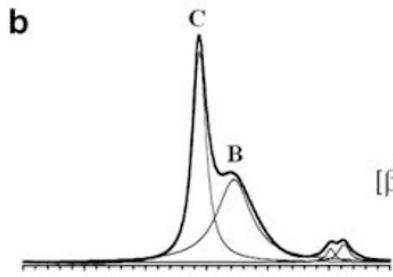

C

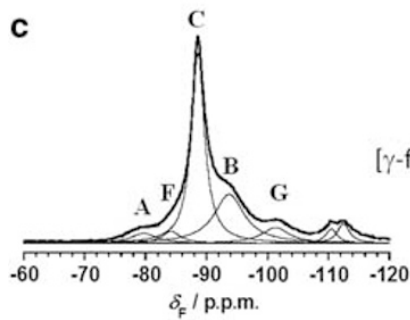

f

e

[ -form]
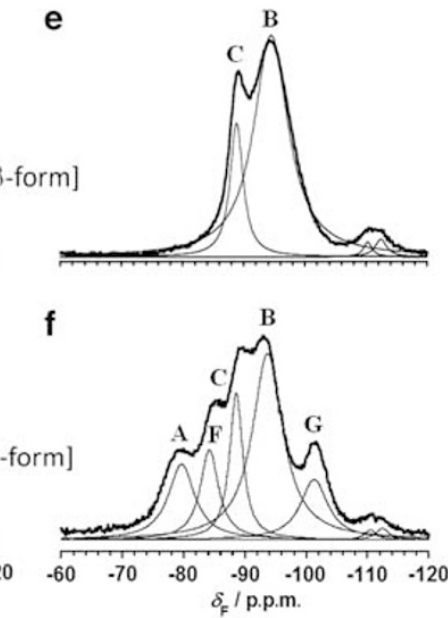

Figure 1 Solid-state ${ }^{19} \mathrm{~F}$ MAS NMR spectra of (a), (d) $\alpha$-powder, (b), (e) $\beta$ film and (c), (f) $\gamma$-film of PVDF. The spectra (a)-(c) were observed using a direct polarization (DP) pulse sequence with broadband ${ }^{1} \mathrm{H}$ decoupling (DP), and the spectra (d)-(f) were observed with the crystalline-selective pulse sequence with spin-locking for $20 \mathrm{~ms}$.

to the amorphous domain, and those at $\delta_{\mathrm{F}}=-110.5$ (D) and -112.5 p.p.m. (E) are assigned to the regio-irregular structures. ${ }^{4}$ In the SL spectrum (Figure 1d), the resonances from the crystalline components (peaks A and B) with longer $T_{1 \rho} \mathrm{F}$, are selectively enhanced, although the residual signals from the amorphous (C) and regio-irregular components $(\mathrm{D}, \mathrm{E})$ are still observed. When compared with the spectra of the $\alpha$-powder, peak A is not observed for the $\beta$-film (Figures $1 \mathrm{~b}$ and $\mathrm{e}$ ), whereas two resonances at higher ( -84.2 p.p.m.) (F) and lower (-101.3 p.p.m.) (G) frequencies are additionally observed for the $\gamma$-film (Figures $1 \mathrm{c}$ and $\mathrm{f}$ ). The peaks of $\mathrm{A}, \mathrm{B}, \mathrm{F}$ and $\mathrm{G}$ have been attributed to the crystalline domains based on the SL spectra (Figure 1f). Because pure $\gamma$-form crystallites would give four equally intense peaks, ${ }^{19}$ the larger intensity of the $\mathrm{B}$ peak compared with peaks A, F and G indicate that a certain amount of $\beta$ form crystallites is incorporated into the $\gamma$-film.

To understand the origin of the characteristic peaks for the three crystalline polymorphs, ${ }^{19} \mathrm{~F}$ nuclear magnetic shieldings are calculated for a model compound consisting of an 8-mer sequence of VDF, $\mathrm{CH}_{3} \mathrm{CF}_{2}-\left(\mathrm{CH}_{2}-\mathrm{CF}_{2}\right)_{7}-\mathrm{CH}_{3}$. Figure 2 shows the stick spectra of ${ }^{19} \mathrm{~F}$ shieldings calculated for $\alpha$-, $\beta$ - and $\gamma$-forms. Figure 3 shows the geometries of the model having the three forms, which were optimized by the DFT calculation. The lattice constants along the polymer chain axis for $\alpha$-, $\beta$ - and $\gamma$-forms have been reported as $4.62 \AA^{1}, 2.56 \AA^{4}$ and $9.18 \AA^{2}$, and those estimated from the optimized geometries are $4.47 \AA$, $2.53 \AA$ and $9.14 \AA$, respectively. The molecular structure of the PVDF chains in each form was well reproduced within errors of $0.4-3.2 \%$. As explained by Holstein et al., ${ }^{15,16}$ two fluorines bonded to the same carbon in the $\alpha$-form are nonequivalent because of its $\mathrm{tg}^{+} \mathrm{tg}^{-}$conformation. In Figure 3, interatomic distances shorter than $3.2 \AA$ are displayed for 1 ) fluorine (F)

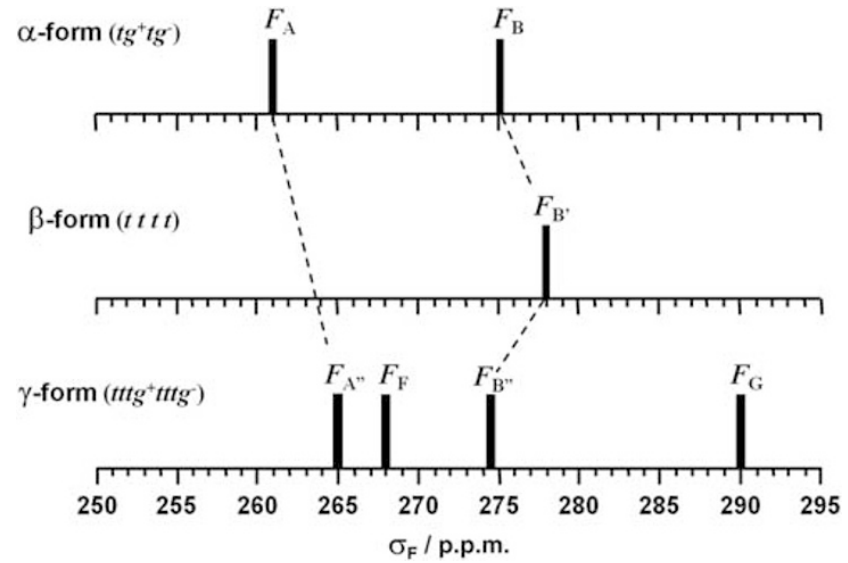

Figure 2 Stick spectra of the ${ }^{19} \mathrm{~F}$ nuclear magnetic shielding calculated for an 8-mer model of PVDF. The molecular geometries were optimized prior to magnetic shielding calculations (see Figure 3 ). Three skeletal conformations of $\mathrm{tg}^{+} \mathrm{tg}^{-}, \mathrm{tttt}, \mathrm{ttg}^{+} \mathrm{tttg}^{-}$were adopted for the VDF main chains with $\alpha-, \beta$ - and $\gamma$-forms, respectively. The sticks $\mathrm{A}, \mathrm{B}, \mathrm{C}, \mathrm{F}$ and $\mathrm{G}$ represent the resonances for fluorines $F_{\mathrm{A}}, F_{\mathrm{B}}, F_{\mathrm{C}}, F_{\mathrm{F}}$ and $F_{\mathrm{G}}$, depicted in Figure 3 , respectively.
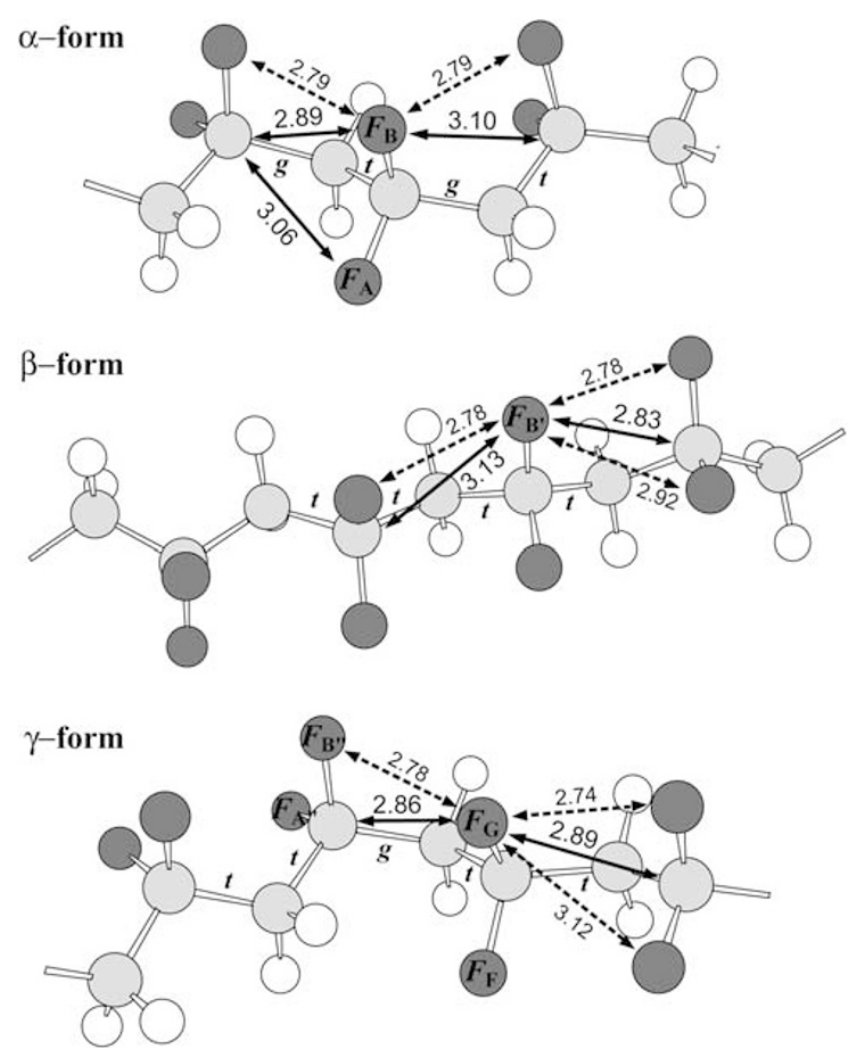

Figure 3 Optimized structures of PVDF models, corresponding to three representative crystalline polymorphs ( $\alpha-, \beta$ - and $\gamma$-forms). Fluorine, carbon and hydrogen atoms are colored black, gray and white, respectively. Inter-atomic distances $(\AA)$ shorter than $3.2 \AA$ are shown for pairs of fluorine and carbon atoms in the $\gamma$-position (solid lines) and fluorine and fluorine atoms in the $\delta$-position (dotted lines). Inter-atomic interactions cause significant shielding effects (low frequency shifts) for fluorine atoms. 
and carbon (C) pairs located in the $\gamma$-position (connected through three covalent bonds and indicated by solid lines) and 2) F pairs located in the $\delta$-position (connected through four covalent bonds and indicated by dotted lines). It is well known that these inter-atomic interactions cause magnetic shielding effects, that is, low frequency shifts of both $\mathrm{F}$ and $\mathrm{C}$ atoms. In the $\alpha$-form, the fluorine $\boldsymbol{F}_{\mathrm{A}}$ is spatially close to only one $\gamma$-gauche carbon without adjacent fluorines in the $\delta$-position, whereas $\boldsymbol{F}_{\mathrm{B}}$ is spatially close to two $\gamma$-gauche carbons and two $\delta$-fluorines. Therefore, the difference in the total amount of ' $\gamma$-gauche interactions' between $\mathrm{C}$ and $\mathrm{F}$ atoms and the ' $\delta$ interactions' between two $\mathrm{F}$ atoms causes a significant chemical shift (14.3 p.p.m.) between $\boldsymbol{F}_{\mathrm{A}}$ and $\boldsymbol{F}_{\mathrm{B}}$. In contrast, all fluorines in the alltrans $\beta$-form $\left(\boldsymbol{F}_{\mathrm{B}}\right.$ ) are equivalent and have two $\gamma$-gauche interactions with carbons and three $\delta$-interactions with fluorines. One additional $\delta$-interaction, compared with $F_{\mathrm{B}}$ in the $\alpha$-form, causes a slightly larger shielding of the $\boldsymbol{F}_{\mathrm{B}}$ : Furthermore, four types of fluorines $\left(\boldsymbol{F}_{\mathrm{A}^{\prime \prime}}, \boldsymbol{F}_{\mathrm{B}^{\prime \prime}}, \boldsymbol{F}_{\mathrm{G}}\right.$, and $\left.\boldsymbol{F}_{\mathrm{F}}\right)$ in the $\gamma$-form, having the $\mathrm{ttg}^{+} \mathrm{tttg}^{-}$conformation, are nonequivalent, and each fluorine exhibits different shielding, as shown in Figure 2. In particular, $\boldsymbol{F}_{\mathrm{G}}$ shows a characteristic resonance at the lowest frequency, that is, the largest shielding, except for the regioirregular components. As shown in Figure $3, \boldsymbol{F}_{\mathrm{G}}$ is adjacent to two $\gamma$ gauche carbons and three $\delta$-fluorines, which is equivalent to $F_{\mathrm{B}}$, in the $\beta$-form. It should be noted, however, that the inter-atomic distances between $\boldsymbol{F}_{\mathrm{B}}$, and its two $\gamma$-gauche carbons are short $(2.83 \AA)$ and slightly long ( $3.13 \AA$ ), whereas those between $\boldsymbol{F}_{\mathrm{G}}$ and its two $\gamma$-gauche carbons are both short $(2.86 \AA$ and $2.89 \AA)$. The superposition of two strong $\gamma$-gauche effects endows $\boldsymbol{F}_{\mathrm{G}}$ with the most shielded resonance. As demonstrated by the experimental and calculated spectra, peak $G$ (the resonance of $\boldsymbol{F}_{\mathrm{G}}$ ) is a characteristic resonance of PVDF $\gamma$-form.

\section{PVDF crystalline structures in PVDF/PMMA blends}

Figure 4 shows the ${ }^{19} \mathrm{~F}$ MAS spectra of blends of [100/0], [80/20] and [70/30]. The spectral shape of these samples in Figures 4a-c (DP spectra) are similar to each other, regardless of the weight fraction of PVDF/PMMA. In addition, the spectral shapes of the crystallineselective SL spectra are similar to one another, as shown in Figures 4d-f. As reported previously, ${ }^{6,7}$ PVDF and PMMA are not fully compatible when the PVDF fraction is high and that PVDF crystallizes in blends of [80/20] and [70/30]. Based on the spectral shapes exhibiting nearly equal intensities of peaks $\mathrm{A}$ and $\mathrm{C}$, the major crystalline structure of PVDF in [100/0], [80/20] and [70/30] is readily characterized as the $\alpha$-form. The degrees of crystallinity $\left(D_{\mathrm{c}}\right)$ estimated from the relative signal intensities, including spinning sideband signals, are estimated as $50 \%, 50 \%$ and $43 \%$ for [100/0], [80/20] and [70/30], respectively. This indicates that the blending of $20 \mathrm{wt} \%$ of PMMA into PVDF does not affect the crystalline structure and the $D_{\mathrm{c}}$ value, although the blending of $30 \mathrm{wt} \%$ PMMA slightly lowers the $D_{c}$. In addition, the slightly larger signal of peak $\mathrm{B}$ than peak $A$ in Figure $4 \mathrm{f}$ suggests that a small amount of $\beta$-form crystallites is generated in the [70/30] sample.

Figure 5 shows the ${ }^{19} \mathrm{~F}$ MAS DP and SL spectra of [65/35], [60/40], [55/45] and [50/50] blends. The spectral shape of (a) and (b) appear different from that of Figure $4 c$, indicating that a drastic alteration in the crystalline structure occurs between [70/30] and [65/35]. Peak A is nearly negligible in the DP spectra of (a) and (b), and the signal intensities of peak B are more than 12 times larger than those of peak $\mathrm{A}$ in the SL spectra of (e) and (f). Therefore, the major crystalline structure of PVDF in [65/35] and [60/40] can be characterized as the $\beta$-form. This partly agrees with the previous report by Horinabe and $\mathrm{Baba}^{7}$ in which $\beta$-form crystallites were detected by wide-angle X-ray diffraction in [80/20], [70/30] and [60/40] blends, which were
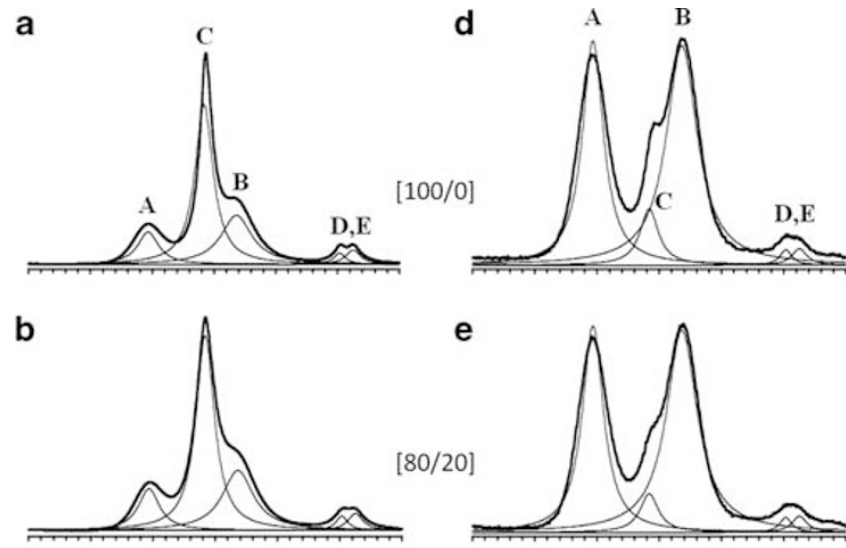

e

$[80 / 20]$
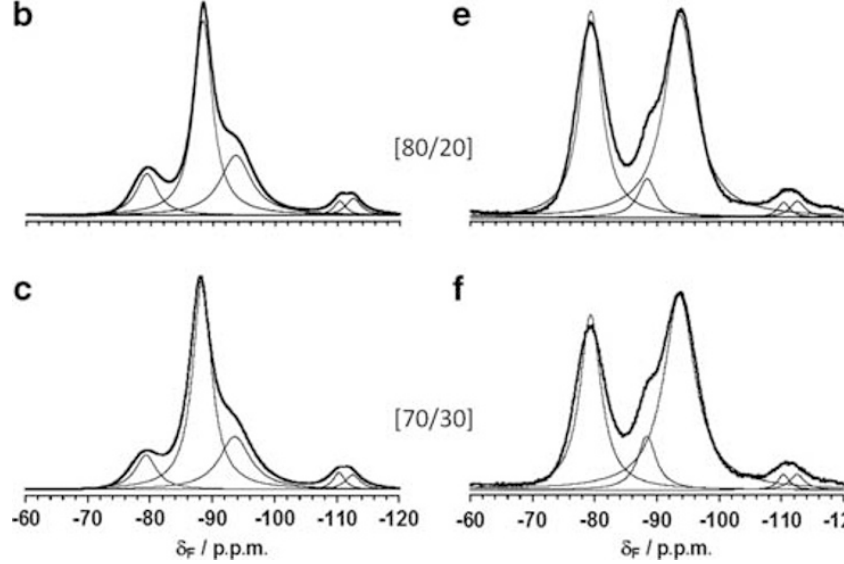

f

Figure $4^{19} \mathrm{~F}$ MAS spectra of PVDF/PMMA blends of [100/0], [80/20] and [70/30]. The spectra of (a)-(c) were observed under direct polarization (DP), and those of (d)-(f) were observed using a crystalline-selective spin-lock pulse sequence. Each spectrum is fitted with a combination of Lorentzian broadening functions (see text).

prepared by quenching from the melt followed by annealing at $120^{\circ} \mathrm{C}$. However, the full width at half maximum of the central wide peak including peaks $\mathrm{C}, \mathrm{B}$ and $\mathrm{G}$ in (e) (9.2 p.p.m.) is 1.5 times as broad as that of the corresponding peak in [70/30] (6.0 p.p.m.) (Figure 4f), and a new component (peak G) is clearly detected at a lower frequency ( -101.3 p.p.m.) in the SL spectra of [65/35] and [60/ 40]. As explained in the previous section, peak $G$ is a characteristic resonance of PVDF $\gamma$-form. Thus, these spectra indicate that the $\beta$-form conformation of PVDF chains in the blends is disordered by the inclusion of a considerable amount of gauche conformation, which leads to the generation of $\gamma$-form crystallites with a $\mathrm{ttg}^{+} \mathrm{tttg}^{-}$ conformation. As a result, the crystalline structure of [65/35] and [60/ $40]$ is characterized as a mixture of both the $\beta$-form and $\gamma$-form, although the former is the dominant structure. However, quantitative estimation of each $\beta$ - and $\gamma$-form was difficult due to the significant overlap of the spectral components. The $D_{\mathrm{c}}$ values estimated from these spectra including both the $\beta$ - and $\gamma$-forms are $41 \%$ for $[65 / 35]$ and $39 \%$ for [60/40]. The crystalline content of PVDF gradually decreases with the increasing PMMA fraction.

Furthermore, the spectral shapes of [55/45] and [50/50] blends are clearly different from those of [65/35] and [60/40]. The signal intensity of peak B is ca. 13 times as strong as that of peak A for [65/35], whereas that is only ca. 4 times stronger for [55/45]. The crystalline contents of $\alpha$ - and $\beta$-forms are $14.5 \%$ and $21.5 \%$, respectively. In addition, the full width at half maximum of peak $B$ is 1.5 times as broad as that of peak $A$, which suggests that the $\beta$-form crystallites in [55/45] still have a broader conformation distribution. Note that the shapes of the DP and SL spectra of [50/50] are very similar to those of [70/30] (Figures $4 \mathrm{c}$ and $\mathrm{f}$ ), indicating that the major crystalline structure in [50/50] is characterized as the $\alpha$-form. Horinabe and $\mathrm{Baba}^{7}$ suggested that the crystalline structure of PVDF 
a
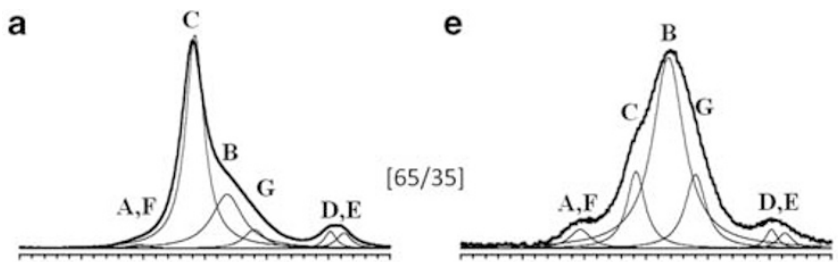

b

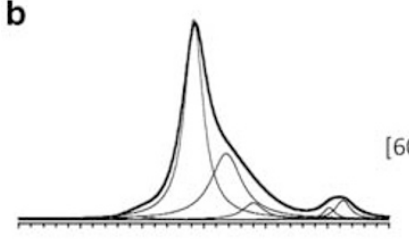

f

C

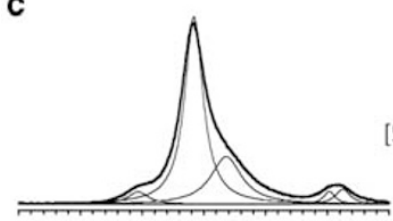

$[55 / 45]$

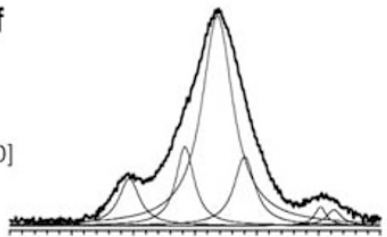

g

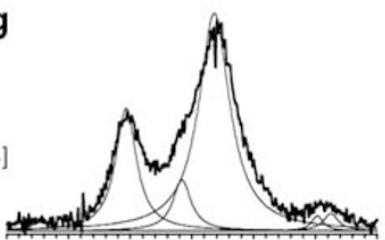

d

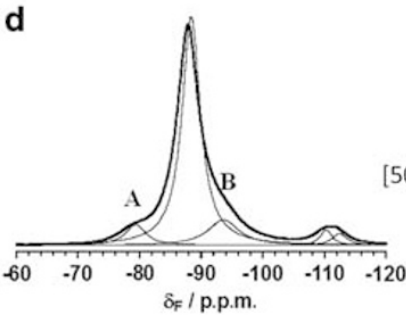

h

$[50 / 50]$

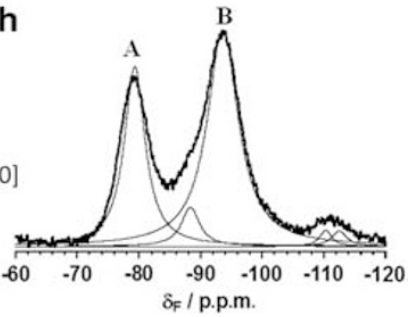

Figure $5{ }^{19} \mathrm{~F}$ MAS spectra of PVDF/PMMA blends of [65/35], [60/40], [55/ 45] and [50/50]. The spectra of (a)-(d) were observed by the DP sequence, and those of (e)-(h) were observed by the crystalline-selective SL sequence.

chains in PVDF/PMMA blends is influenced by the rate of crystallization. ${ }^{7}$ For the case of relatively slow crystallization, PVDF preferentially forms $\alpha$-form. The formation of $\alpha$-form in a [50/50] blend can be attributed to the relatively slow crystallization at lower PVDF fractions and the good miscibility of PVDF and PMMA chains in the blend, which is supported by the lower $D_{c}$ value $(26 \%)$ of the [50/50] blend. This fact also indicates that the all-trans $\beta$-form and trans-rich $\gamma$-form are preferentially generated only in a certain range of PVDF fractions for the case that both the PVDF fraction and the rate of crystallization meet appropriate conditions.

In contrast to the blended films analyzed above, Figures $6 a-c$ demonstrates that no crystalline signals are observed in the DP spectra of [45/55], [40/60] and [20/80]. This finding clearly indicates that PVDF and PMMA are fully compatible on the molecular level and that PVDF chains are completely included in the amorphous phase. This fact is supported by the good optical transparency of these films, whereas the semi-crystalline blend films at the higher PVDF fractions (50\%) look slightly translucent owing to visible light scattering. Although the DP spectra of these blends appear similar to each other, the values of the full width at half maximum for the main peaks are 4.7, 5.7 and 6.9 p.p.m. for [45/55], [40/60] and [20/80], respectively. The gradual increase in spectral width indicates that the main chain conformations have wider distributions at decreasing PVDF fractions. Because of the high glass transition temperature $\left(T_{\mathrm{g}}\right)$ of PMMA $\left(122^{\circ} \mathrm{C}\right)$, the $T_{\mathrm{g}} \mathrm{s}$ of the miscible blends approach to that of PMMA and become higher than the
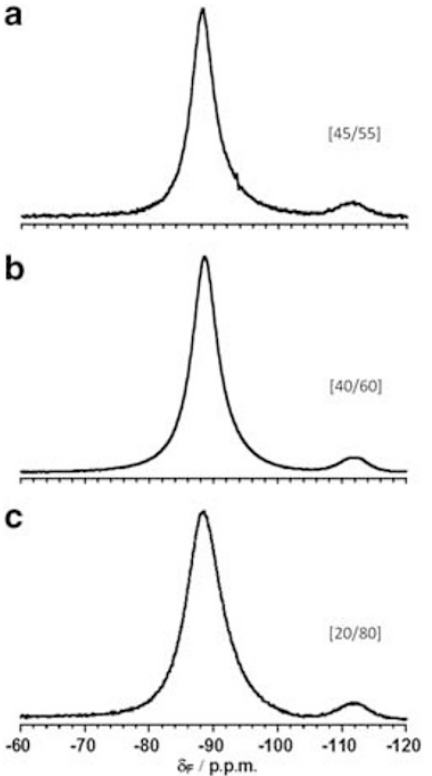

Figure $6{ }^{19} \mathrm{~F}$ MAS spectra of PVDF/PMMA blends of [45/55], [40/60] and [20/80]. All the spectra were observed under DP sequence, and no signals were observed by the $S L$ sequence. The full widths at half maximum of the main peaks are 4.6, 5.8 and 6.9 p.p.m. for (a), (b) and (c), respectively.

measurement temperature with decreasing PVDF fraction. The restricted motion of the PVDF chains in the glassy state should lead to increases in the spectral width.

Figure 7 summarizes the degrees of crystallinity $\left(D_{\mathrm{c}}\right)$ of PVDF chains in the blend films, together with the crystalline contents of the $\alpha$-, $\beta$ - and $\beta+\gamma$-forms. As mentioned above, the $D_{c}$ values gradually decrease with the increase in PMMA fraction from [80/20] to [50/50]. Assuming that the PVDF content in the amorphous phase for [70/ 30]-[50/50] are close to each other based on 'the lever rule', the gradual decrease in $D_{\mathrm{c}}$ values is reasonably related to the total amount of PMMA in the blends. Meanwhile, the crystalline $\alpha$-form having a $\mathrm{tg}^{+} \mathrm{tg}^{-}$conformation is dominant in [100/0], [80/20] and [70/30], whereas all-trans $\beta$-form is dominant in [65/35], [60/40] and [55/45] with wider distributions in chain conformation. This is caused by the incorporation of certain amounts of $\gamma$-form having a $\mathrm{ttg}^{+} \mathrm{tttg}^{-}$ conformation. Although $\alpha$-form appears again as the major crystalline structure in [50/50], PVDF is completely compatible with PMMA at lower PVDF fractions ([45/55], [40/60] and [20/80]). All PVDF chains are incorporated in the amorphous phase without crystalline morphology in these blends.

\section{Molecular mobility of the amorphous phase}

The ${ }^{19} \mathrm{~F}$ MAS NMR measurements with variable SL times were performed to investigate the molecular mobility of ${ }^{19} \mathrm{~F}$ nuclei in the blends. Figure 8 shows the variations in the ${ }^{19} \mathrm{~F}$ spin-lattice relaxation time in the rotating frame $\left(T_{1 \rho}{ }^{\mathrm{F}}\right)$ observed for the amorphous signal $\mathrm{C}\left(-88.7\right.$ p.p.m.) and the $T_{\mathrm{g}} \mathrm{s}$ for the blends with different PVDF fractions. For the PVDF homopolymer [100/0], the $\alpha$-relaxation temperature estimated by dynamic modulus spectroscopy was adopted as the $T_{\mathrm{g}}$ value because the glass transition was not clearly detected by DSC. In addition, the $T_{\mathrm{g}}$ of [80/20] is not plotted because the glass transition was not detected by DSC and dynamic modulus spectroscopy. Note that the $T_{\mathrm{g}}$ is significantly elevated from $-37^{\circ} \mathrm{C}$ to $+52^{\circ} \mathrm{C}$ by blending $30 \mathrm{wt} \%$ of PMMA into 


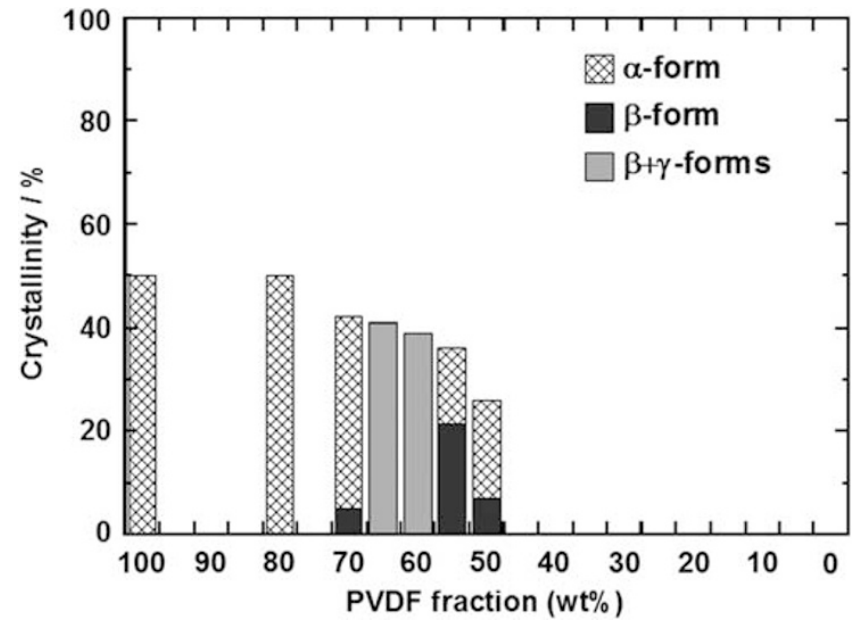

Figure 7 Degree of crystallinity $\left(D_{c}\right)$ versus weight fraction of PVDF together with the crystalline contents of the $\alpha-, \beta$ - and $\beta+\gamma$-forms in the PVDF/ PMMA blends.

a
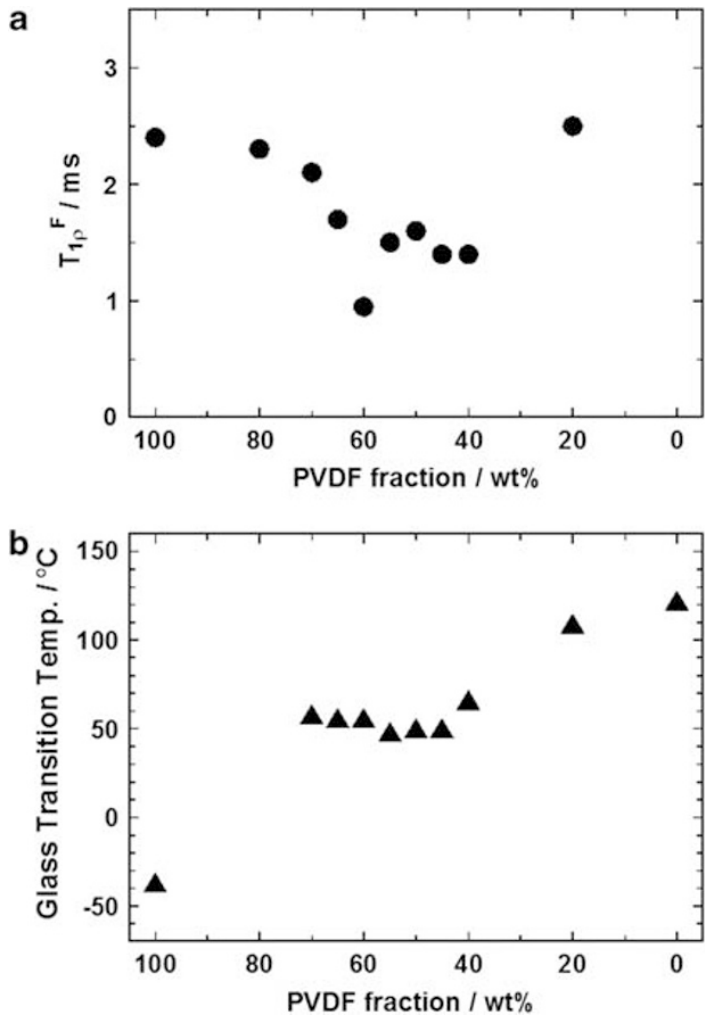

Figure 8 (a) ${ }^{19} \mathrm{~F}$ spin-lattice relaxation times in the rotating frame $\left(T_{1 p}{ }^{F}\right)$ observed for the amorphous signal (peak $\mathrm{C}$ in Figure 1) and (b) glass transition temperatures $\left({ }^{\circ} \mathrm{C}\right)$ measured with DSC versus weight fractions of PVDF (wt\%) in the PVDF/PMMA blends.

neat PVDF and that the $T_{1 p} \mathrm{~F}$ values for the amorphous phase gradually decreased, as shown in Figure $8 \mathrm{a}$. A minimum is found for the $T_{1 \rho}{ }^{\mathrm{F}}$ of [60/40]. Based on the theory of nuclear magnetic relaxation, ${ }^{24}$ the molecular motion of PVDF main chain in the amorphous phase is close to the SL frequency of $\sim 83 \mathrm{kHz}$ at approximately this PVDF fraction. For blends with higher fractions
( $>60 \%$ ) of PVDF, the $T_{1 \rho}{ }^{\mathrm{F}}$ values exhibit rubber-like behavior, which is because the $T_{\mathrm{g}} \mathrm{s}$ of these blends is lower than the measurement temperature $\left(68^{\circ} \mathrm{C}\right)$, as depicted in Figure $8 \mathrm{~b}$. The unchanged $T_{\mathrm{g}}$ values (approximately $55^{\circ} \mathrm{C}$ ) observed for blends between [55/45] and [40/60] agree well with their stable $T_{1 \rho}{ }^{\mathrm{F}}$ values at approximately $1.5 \mathrm{~ms}$. These facts indicate that the mobility of PVDF in the amorphous phase are not significantly influenced by polymorphs in the crystalline phase. Furthermore, the $T_{1 \rho}{ }^{\mathrm{F}}$ value again increases for [20/80] with the lower PVDF fraction, which demonstrates that the molecular motion of the PVDF chains is restricted as the minor component in the blend. This is also reflected in the fact that the $T_{\mathrm{g}}$ $\left(108^{\circ} \mathrm{C}\right)$ of $[20 / 80]$ goes over the measuring temperature owing to the high PMMA fraction ( $80 \mathrm{wt} \%)$. The variations in $T_{1 \rho}{ }^{\mathrm{F}}$ partly agree with the gradual increases in spectral widths of the amorphous signals, as discussed above.

\section{CONCLUSION}

The origin of the characteristic resonances in typical polymorphs of $\operatorname{PVDF}(\alpha-, \beta$ - and $\gamma$-forms) is explained based on the DFT magnetic shielding calculation of an 8-mer chain of PVDF. The steric effects represented by ' $\gamma$-gauche interactions' between $\mathrm{C}$ and $\mathrm{F}$ atoms and the ' $\delta$-interactions' between two $\mathrm{F}$ atoms induce significant low-frequency shifts to fluorine atoms, which are the main cause of the chemicals shifts observed in the polymorphs. The crystalline structure and molecular mobility of PVDF blended with PMMA were investigated by ${ }^{19} \mathrm{~F}$ MAS NMR spectroscopy. The variations in the degree of crystallinity and the major conformations of the crystalline PVDF component in the blends are quantitatively analyzed from the NMR spectral shapes, and the molecular mobility in the amorphous phase is inferred based on their $T_{1 \rho}{ }^{\mathrm{F}}$ values. The dominant crystalline form in [100/0] [80/20], [70/20] and [50/50] is the $\alpha$-form with a $\operatorname{tg}^{+} \mathrm{tg}^{-}$ conformation, whereas blends of [65/35], [60/40] and [55/45] have a $\beta$-form containing a certain amount of the $\gamma$-form. $\beta$-form has an all-trans conformation, and the $\gamma$-form has a $\mathrm{ttg}^{+} \mathrm{tttg}^{-}$conformation, which indicates that the PVDF chains possess more elongated conformations along the polymer chains in these blends. In contrast, no crystalline structure is found in [45/55], [40/ 60] and [20/80], indicating that PVDF and PMMA chains are miscible on the molecular level. The $T_{1 \rho} \mathrm{F}$ values estimated for the amorphous phase are typical for the rubbery state with the minimum observed for the [60/40] blend, indicating that the mobility of the PVDF chains in the amorphous phase is close to the SL frequency $(\sim 83 \mathrm{kHz})$. The larger $T_{1 \rho}{ }^{\mathrm{F}}$ value observed for [20/80] agrees with that the fact that the $T_{\mathrm{g}}\left(108^{\circ} \mathrm{C}\right)$ of the blend was higher than the measuring temperature $\left(68^{\circ} \mathrm{C}\right)$. Solid-state ${ }^{19} \mathrm{~F}$ MAS NMR provides unequivocal insights into the molecular structure and mobility of PVDF chains, which are miscible with PMMA at the molecular level.

1 Hasegawa, R., Kobayashi, M. \& Tadokoro, H. Molecular conformation and packing of poly(vinylidene fluoride). Stability of three crystalline forms and the effect of high pressure. Polym. J. 3, 591-599 (1972).

2 Matsushige, K. \& Takemura, T. Melting and crystallization of poly(vinylidene fluride) under high pressure. J. Polym. Sci.: Polym. Phys. 16, 921-934 (1978).

3 Bachmann, M. A. \& Lando, J. B. A. Reexamination of the Crystal Structure of Phase II of Poly(vinylidene fluoride). Macromolecules 14, 40-46 (1980).

4 Takahashi, Y., Matsubara, Y. \& Tadokoro, H. Mechanisms for crystal phase transformations by heat treatment and molecular motion in poly(vinylidene fluoride). Macromolecules 15, 334-338 (1982).

5 Nishi, T. \& Wang, T. T. Melting point depression and kinetic effects of cooling on crystallization in poly(vinylidene fluoride)-poly(methyl methacrylate) mixtures. Macromolecules 8, 909-915 (1975). 
6 Wang, T. T. \& Nishi, T. Spherulitic crystallization in compatible blends of poly(vinylidene fluoride) and poly(methyl methacrylate). Macromolecules 10, 421-425 (1977).

7 Horinabe, H. \& Baba, F. Changes in crystal structures of PVDF in PVDF/PMMA blends heat-treated in several ways. Nippon Kagaku Kaishi 121-126 (2000).

8 Coleman, M. M., Zarian, J., Varnell, D. F. \& Painter, P. C. A. Fourier transform infrared study of polymer blends. I. Poly(vinylidene fluoride)/poly(methyl methacrylate) system. J. Polym. Sci.: Polym. Lett. 15, 745-750 (1977).

9 Hahn, B. R., Herrmann-Schonherr, O. \& Wendorff, J. H. Evidence for a crystalamorphous interphase in PVDF and PVDF/PMMA blends. Polymer 28, 201-208 (1987).

10 Douwel, C. H. K., Maas, W. E. J. R. \& Veeman, W. S. Miscibility in PMMA poly(vinylidene fluoride) blends, studied by fluorine-19-enhanced carbon 13 CPMAS NMR. Macromolecules 23, 406-412 (1990).

11 Papavoine, C. H. M., Maas, W. E. J. R. \& Veeman, W. S. Triple-resonance protonfluorine-19-carbon-13 CPMAS NMR study on the crystallization behavior of the PMMA/ poly(vinylidene fluoride) 60/40 blend. Macromolecules 26, 6611-6616 (1993).

12 Harris, R. K. \& Jackson, P. High-resolution fluorine-19 magnetic resonance of solids. Chem. Rev. 91, 1427-1440 (1991).

13 Miller, J. M. Fluorine-19 magic-angle spinning NMR. Prog. NMR Spectrosc. 28, 255-281 (1996).

14 Scheler, U. \& Harris, R. K. Proton-fluorine wideline separation experiments on poly(vinylidene difluoride). Solid State Nucl. Magn. Reson. 7, 11-16 (1996).

15 Holstein, P., Harris, R. K. \& Say, B. J. Solid-state ${ }^{19} \mathrm{~F}$ NMR investigation of poly(vinylidene fluoride) with high-power proton decoupling. Solid State Nucl. Magn. Reson. 8, 201-206 (1997).
16 Holstein, P., Scheler, U. \& Harris, R. K. Semicrystallinity and polymorphism in PVDF: A solid-state ${ }^{19}$ F n.m.r. investigation. Polymer 39, 4937-4941 (1998).

17 Ando, S., Harris, R. K. \& Reinsberg, S. A. Solid-state ${ }^{1} \mathrm{H} \rightarrow{ }^{19} \mathrm{~F} /{ }^{19} \mathrm{~F} \rightarrow{ }^{1} \mathrm{H}$ CP/MAS NMR study of poly(vinylidene fluoride). Magn. Reson. Chem. 40, 97-106 (2002).

18 Ando, S., Harris, R. K. \& Scheler, U. Supplement of encyclopedia nuclear magnetic resonance ChichesterJohn Wiley \& Sons, 2002).

19 Tatsuno, H., Koseki, Y. \& Ando, S. Structure and dynamics of a vinylidene fluoride oligomer and its cyclodextrin inclusion compounds as studied by solid-state ${ }^{19} \mathrm{~F}$ MAS and ${ }^{1} \mathrm{H} /{ }^{19} \mathrm{~F}$ CP/MAS NMR spectroscopy. Polymer 49, 2709-2716 (2008).

20 Hucher, C., Beaume, F., Eustache, R.-P. \& Tekely, P. Probing phase structure and location of reverse units in poly(vinylidene fluoride) by solid-state NMR. Macromolecules 38, 1789-1796 (2005).

21 Park, J.-W., Seo, Y.-A., Kim, I. \& Ha, C.-S. Investigating the crystalline structure of poly(vinylidene fluoride) (PVDF) in PVDF/silica binary and PVDF/poly(methyl methacrylate)/silica ternary hybrid composites using FTIR and solid-state ${ }^{19} \mathrm{~F}$ MAS NMR spectroscopy. Macromolecules 37, 429-436 (2004).

22 Saito, H. \& Inoue, T. Chain orientation and intrinsic anisotropy in birefringence-free polymer blends. J. Polym. Sci. B: Polym. Phys. 25, 1629-1636 (1987).

23 Vierkötter, S. A. Applications of the Bloch-Siegert shift in solid-state protondipolar-decoupled 19F MAS NMR. J. Magn. Reson. Series A 118, 84-93 (1996).

24 Bloembergen, E. M., Purcell, R. V. \& Pound, R. Relaxation effects in nuclear magnetic resonance absorption. Phys. Rev. 73, 679-746 (1948). 